\section{P26 (continued)}

benefits. Narrative analysis assessed information from preand post-surveys, as well as observations and experiences of participants.

Results: The pre- and post-survey assessment revealed children had higher awareness of the Health360 policies after attending the lessons. All participants believed in the importance of embodying the policies post-lessons, as opposed to $25 \%$ pre-instruction. It was observed that the more lessons attended, the more children were engaged in learning about healthy habits. When a health policy was taught a second time it reinforced the lesson and participants retained information. They asked insightful questions, grasped the benefits of healthful lifestyles, and contemplated making changes in their lives.

Conclusion: Childhood obesity is a major health concern, which is exacerbated by a lack of positive examples for healthy habits. When children are exposed to the benefits of healthy lifestyles they are more likely to make changes. This could aid in reducing the risk of chronic disease.

Funding: None.

\section{P27 Health Behavior Changes and Mental Health During the COVID-19 Crisis in Food Insecure College Students}

Alison Macchi, MS, amatt008@fiu.edu, Florida

International University, 11200 SW 8th St, Miami, FL, 33199; Catherine Coccia, PhD, RDN, Florida International University

Background: The COVID-19 pandemic has had impactful and possibly long-term effects on the lives of college students. This may be particularly challenging for food insecure students, who are at a higher risk for negative effects of the COVID-19 pandemic.

Objective: The purpose of this study was to determine the effects of COVID-19 on the health behaviors and mental health of food insecure college students.

Study Design, Setting, Participants: A mixed-methods study design was used to determine the changes in mental and health behaviors in food insecure college students. Students completed 2 surveys over 6 months, pre and post COVID-19. Surveys were composed of validated measures and open-ended questions. Participants were asked to describe their demographics, food security, fruit and vegetable intake, stress, and life satisfaction. Food insecure students $(n=41)$ were recruited from Florida International University. Participants were female (92.5\%), White Caucasian (42.5\%), and Hispanic (60\%), and of normal BMI (52.5\%).

Measurable Outcome/Analysis: Statistical analysis was performed using SPSS v26. Descriptive statistics, frequencies, and $t$ tests were performed on validated measures. Diet and physical activity open response questions were categorized into positive, negative or neutral changes due to COVID-19. Overall changes went through a thematic review and were categorized into themes.

Results: Post COVID-19, participants significantly decreased their fruit and vegetable intake $(t=-2.12, P=$ 0.04). Participants indicated both positive and negative changes in diet and physical activity. Participants also increased their perceived stress $(t=2.37, P=0.02)$ and decreased life satisfaction $(t=-3.03, P<0.01)$ but indicated positive changes including getting closer to family and friends, improved work, school, and finances, and had increased time to enjoy activities.

Conclusion: COVID-19 had both positive and detrimental effects on diet, physical activity, stress and life satisfaction of food insecure students. While overall measures declined, students indicated that quarantine gave them an opportunity to work on personal relationships and mental health.

Funding: None.

\section{P28 Herbs and Spices Found in a Grocery Store Where Low-Income Mexicans Frequently Shop}

Graham E.Bastian, RDN, g.e.bastian@rutgers.edu, Rutgers University, Department of Nutritional Sciences, 26 Nichol Ave, New Brunswick, NJ, 08902; Ricardo Kairios, BS, Rutgers University, Department of Nutritional Sciences; Sebastian Tapia, Rutgers University, Department of Nutritional Sciences; Debra M. Palmer-Keenan, PhD, MEd, Rutgers University, Department of Nutritional Sciences

Background: Since herbs, spices, spice blends, and salts $(H \& S)$ vary by culture, it is imperative for recipes created for specific audiences to include H\&S that are both culturally relevant and readily available. Yet, lists of $H \& S$ used by immigrants residing in various regions of the U.S. do not exist.

Objective: To document and investigate the H\&S available to low-income Mexicans and Mexican-Americans in a small, urban NJ community.

Study Design, Setting, Participants: This was a descriptive study of $H \& S$ available in the largest grocery store frequented by the Mexican community in New Brunswick, NJ. With the store manager's permission, photographs were taken and used to document all H\&S in the store.

Measurable Outcome/Analysis: The names of all H\&S in the photographs that did not appear on a previously developed list of H\&S commonly used in the U.S. were recorded and translated into English by a bilingual research assistant (when necessary). An internet search was conducted to determine the H\&S's common use among Mexicans and other Latinos.

Results: Forty-five H\&S that did not appear on the previously developed list of H\&S used in the U.S. were identified. Notably, $21 \mathrm{H} \& S$ were not used for culinary purposes, but instead had a wide range of functional and medicinal purposes ranging from weight loss to combating parasites. 


\section{P28 (continued)}

Conclusion: The researchers, despite being experienced nutrition educators, were surprised at the number of unfamiliar H\&S, and that many were used for medicinal purposes yet were grouped with $H \& S$ used for culinary purposes, sometimes even on the same shelf. These findings support the following implications: 1) inclusion of community members in nutrition education materials development is essential for cultural relevance; 2) nutrition educators could benefit from additional cultural cuisine training; and 3) approaching the study of non-Western cultures with an ethnocentric bias may prohibit a thorough understanding of how people may use H\&S at home.

Funding: None.

\section{P29 Interest and Acceptability of a Clinic Based EFNEP Intervention in English and Spanish Speaking Parents for Pediatric Obesity Prevention}

Mical Shilts,PhD, MS, shiltsm@csus.edu, California State University, Sacramento, 6000 Jst, Sacramento, CA, 95819 6053; Karina Diaz Rios, PhD, RDN, University of California, Merced; Lenna Ontai, $P h D$, University of California, Davis; Katherine Panarella, MPH, MS, University of California, Davis, Agriculture and Natural Resources; Christiana Drake, PhD, University of California, Davis; Dennis Styne, $M D$, University of California, Davis Children's Hospital; Samantha Navarro, BA, University of California, Merced; Adrian Loera, BS, University of California, Merced; Louise Lanoue, PhD, University of California, Davis; Marilyn Townsend, PhD, $R D$, University of California, Davis

Objective: Obesity continues to disproportionately impact low-income, ethnically diverse youth. Existing government-funded nutrition education programs and pediatric medical clinics can collaborate to target and deliver relevant interventions. The objective was to explore interest and acceptability of community nutrition intervention collocated in medical clinics.

Use of Theory: Social Cognitive (SCT) and goal setting theories.

Target Audience: Low-income English and Spanishspeaking parents with young children.

Program Description: Physicians referred parents of pediatric patients to the intervention. The intervention consisted of 8 -weekly, 1.5 hour nutrition, activity and food-related parenting content anchored with guided goal setting, SCT constructs, and story telling. Existing Expanded Food and Nutrition Education (EFNEP) educators delivered the intervention in medical clinics.

Evaluation Methods: To assess interest and acceptability, referral, enrollment, and attendance data were collected and parent focus group interviews were conducted. Descriptive statistics and chi-square analysis were conducted on the quantitative data and interviews were recorded, transcribed and organized for content analysis.
Results: Twenty intervention series were conducted in English $(\mathrm{n}=12)$ and Spanish $(\mathrm{n}=8)$ at 5 medical clinics by 4 EFNEP educators. More than $1 / 3$ of the parents $(37 \% ; n$ = 709) expressed interest in the intervention after physician referral and $74 \%(\mathrm{n}=195)$ enrolled in the intervention. A greater proportion of referred Spanish-speaking parents expressed interest $(P<0.001)$ and completed 4 or more intervention sessions $(P<0.0001)$ compared to English-speaking parents. A total of 26 focus group interviews $(n=65)$ were conducted with the $97 \%$ of the parents said they enjoyed participating in the intervention. The intervention component most commonly mentioned as useful was guided goal setting.

Conclusion: Results from this study indicated that parents referred to the intervention were interested and attended with greater appeal to Spanish-speaking parents. Acceptability of the intervention can be attributed to the tailoring of the content and delivery by culturally competent educators.

Funding: NIFA.

\section{P30 Interface of Neighborhood Residence on Obesity Prevention Behaviors and Self-Efficacy in Chinese Americans}

Doreen Liou, EdD, RDN, lioud@montclair.edu, Montclair State University, 1 Normal Ave, Montclair, NJ, 07043; Jessica Karasik, BA, Montclair State University

Background: The obesity epidemic is a prominent health issue affecting Chinese Americans in the United States. An individual's living environment affects dietary choices and consumption patterns, impacting weightrelated outcomes. The important construct of self-efficacy provides a foundation for nutrition educators in understanding mediators of health behaviors.

Objective: To identify if self-reported neighborhood of residence ('high income' vs 'middle-low income') elucidate differences in obesity prevention behaviors and selfefficacy in Chinese Americans residing in Los Angeles county and the New York metropolitan area.

Study Design, Settings, Participants: A cross-sectional survey design was used where a convenience sample of 650 participants (18-60 years old) completed a validated, self-administered questionnaire. Participants were recruited from universities, cultural institutions, and churches, representing a wide range of backgrounds.

Measurable Outcome and Analysis: Nineteen behaviors related to obesity risk reduction were measured with 9 items measuring self-efficacy for these behaviors. Participants indicated adoption of behaviors over the month reflecting food practices, portion size control, and consumption of plant-based foods. Descriptive statistics were conducted for the entire sample while $t$ tests were applied for categorized neighborhoods ('high income' vs 'middle-low income').

Results: The mean age of the study participants $(67 \%$ 